\title{
A case of levocetirizine-induced liver injury
}

\author{
Moon Chan Jung', Ja Kyung Kim², , Jae Yeon Cho', Jae Won Song', Bohyun Lee', Ji Won Park ${ }^{1,2}$, Jinwon Seo ${ }^{3}$, and \\ Sung Eun $\mathrm{Kim}^{1,2}$ \\ ${ }^{1}$ Department of Internal Medicine, ${ }^{2}$ Division of Gastroenterology, ${ }^{3}$ Department of Pathology, Hallym University Sacred Heart Hospital, \\ Hallym University College of Medicine, Anyang, Korea
}

Levocetirizine is a second-generation nonsedative antihistaminic agent that has been demonstrated to be safe and effective for treating allergic disease. There was only one case report of levocetirizine-induced liver toxicity, but a liver biopsy was not performed. In this article, we present the first case of levocetirizine-induced liver injury with histologic findings. A 48-year-old man was hospitalized with jaundice and generalized pruritus that had developed after 2 months of therapy with levocetirizine for prurigo nodularis. Laboratory findings revealed acute hepatitis with cholestasis. A liver biopsy demonstrated portal inflammation and hepatitis with apoptotic hepatocytes. The patient fully recovered 3 weeks after withdrawing levocetirizine. Although levocetirizine is safe and effective, physicians should be aware of its potential hepatotoxicity. (Clin Mol Hepatol 2016;22:495-498)

Keywords: Hepatotoxicity; Levocetirizine; Cholestasis

\section{INTRODUCTION}

Levocetirizine (LCZ), an active enantiomer of cetirizine, is a second-generation antihistamine that was approved by the US Food and Drug Administration in 2008 for the relief of symptoms associated with allergic diseases in adults and children. ${ }^{1-3}$ Although it has some side effects — such as sleepiness, headache, dry mouth, vision problems, palpitation, and fatigue-it is considered to be effective and safe treating allergic disease. ${ }^{4}$ Only one case of LCZinduced liver injury has been reported. ${ }^{5}$

Here, we present the first report of LCZ-induced liver injury with histologic confirmation in a 48-year-old man without a history of hepatobiliary disease.

\section{CASE REPORT}

A 48-year-old man without significant medical history was referred from the dermatology department due to jaundice and generalized pruritus. The patient did not report any fatigue, anorexia, nausea, abdominal discomfort, fever, rash, myalgia, muscle weakness, swelling, and recent illness. He had no previous history of hepatobiliary disease. The patient's medical history was only notable for prurigo nodularis. Medication in recent use included LCZ 5 mg daily for 2 months, and the patient had been taking bepotastine besilate $10 \mathrm{mg}$ daily until 2 months ago. He did not report taking any other medications including statins, over-the-counter medications, herbal medications including red ginseng or any extract or nutritional supplements. Specific history of exposure to hepatotox-

\section{Abbreviations:}

ALT, alanine aminotransferase; AST, aspartate aminotransferase; DILI, drug-induced liver injury; LCZ, levocetirizine

\section{Corresponding author: Sung Eun Kim}

Department of Internal Medicine, Hallym University Sacred Heart Hostpital, University of Hallym College of Medicine, 22 Gwanpyeong-ro 170beon-gil, Dongan-gu, Anyang 14068, Korea

Tel: +82-31-380-3705, Fax: +82-31-386-2269

E-mail: sekim@hallym.or.kr 

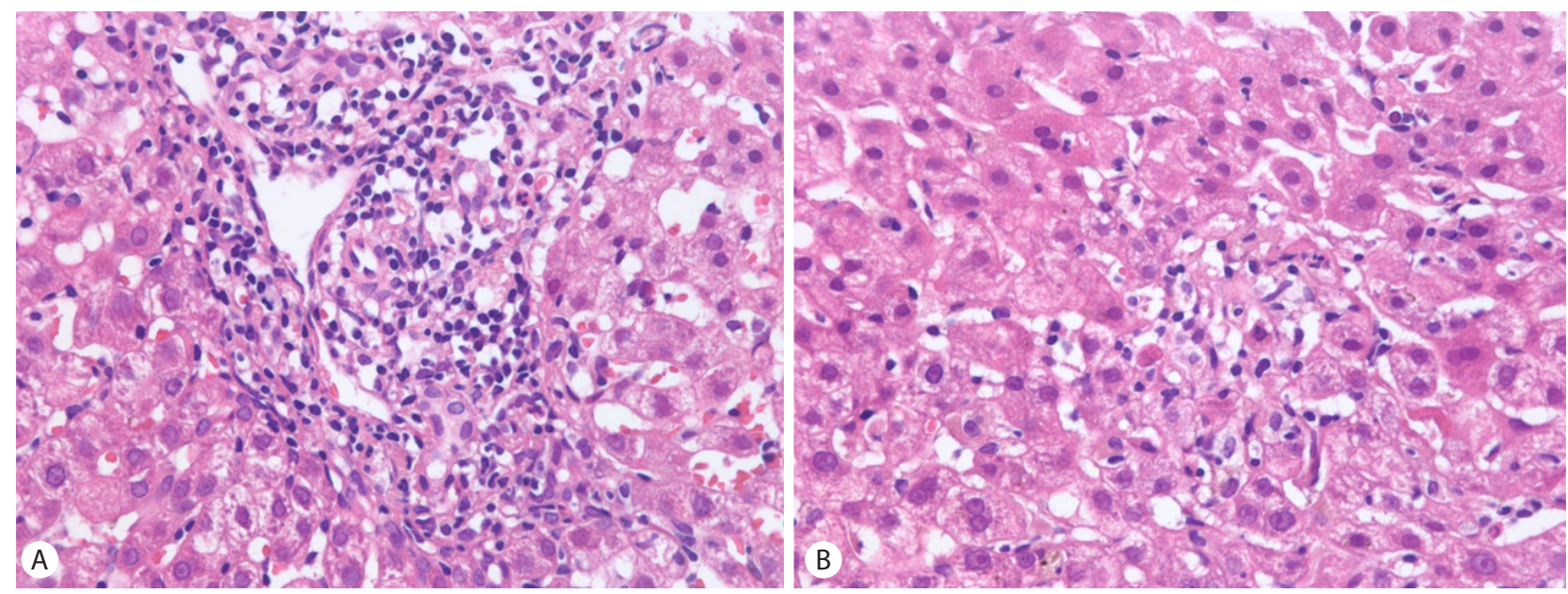

Figure 1. (A) Liver biopsy tissue $(H \& E, \times 200)$ showing patchy portal inflammation and hepatocyte dropout. Portal and periportal inflammation is evident. (B) The hepatic parenchyme also shows multifocal hepatocellular dropouts with apoptotic hepatocytes.

ic chemicals or familial history of liver disease was not identified. He also had no travel history. Alcohol consumption was limited to regular ethanol consumption of $57 \mathrm{~g} /$ week.

Initial blood pressure was $110 / 70 \mathrm{mmHg}$, pulse rate $88 / \mathrm{min}$, respiratory rate $18 / \mathrm{min}$, and body temperature $36.7^{\circ} \mathrm{C}$. Physical examination revealed a jaundice with icteric sclera. He had a soft abdomen without tenderness. He had no hepatomegaly, splenomegaly, and stigmata of chronic liver disease.

Laboratory findings revealed prothrombin time $11.8 \mathrm{~s}$, international normalized ratio 0.88 , aspartate aminotransferase (AST) 1,208 IU/L, alanine aminotransferase (ALT) 2,043 IU/L, total bilirubin $8.68 \mathrm{mg} / \mathrm{dL}$, direct bilirubin $7.23 \mathrm{mg} / \mathrm{dL}$, alkaline phosphatase $184 \mathrm{U} / \mathrm{L}$, lactate dehydrogenase $463 \mathrm{IU} / \mathrm{L}$, gamma-glutamyl transpeptidase $253 \mathrm{IU} / \mathrm{L}$, protein $5.9 \mathrm{~g} / \mathrm{dL}$, albumin $3.9 \mathrm{~g} / \mathrm{dL}$, and creatine phosphokinase $21 \mathrm{IU} / \mathrm{L}$. Serologic viral marker evaluations revealed hepatitis B surface antigen negative, antibody to hepatitis B surface antigen positive, antibody to hepatitis B core antigen negative, anti-hepatitis $C$ virus negative and anti-human immunodeficiency virus negative. Serum anti-mitochondrial antibody, anti-smooth muscle antibody, anti-nuclear antibody, antidsDNA antibody, anti-neutrophil cytoplasm antibody, and anti-liver kidney microsome antibody were negative. Serum ceruloplasmin and copper levels were within normal ranges. His abdominal X-ray showed no specific findings. An abdominal sonography revealed no evidence of extrahepatic obstruction, biliary ductal disease, cholelithiasis, or hepatic parenchymal abnormalities. The size, shape, and echotexture of the liver were normal. For histologic evaluation, a percutaneous ultrasonography-guided liver biopsy was performed without complications. The liver biop- sy showed portal inflammation and hepatitis with apoptotic hepatocytes. There was an increased amount of connective tissue extending from the portal area and inflammation around apoptotic hepatocytes (Fig. 1).

The patient stopped taking LCZ immediately after evaluation and all kinds of liver enzymes were normalized within 3 weeks after discontinuation (Fig. 2). The patient visited the outpatient department several times after discharge and has maintained normal liver function.

\section{DISCUSSION}

Drug-induced liver injury (DILI) is a rare but potentially lifethreatening adverse drug reaction. A large number of drugs have deleterious effects on the liver, and DILI is a major cause of acute liver injury and liver transplantation. ${ }^{6}$ DILI is responsible for $~ 10 \%$ of all adverse drug reactions in the United States. ${ }^{7}$ In Korea, the annual extrapolated incidence of DILI in hospitalized patients at a university hospital was 12/100,000 persons/year. ${ }^{8}$ The mechanism of DILI is unknown, but it is not associated with medication dose, particularly idiosyncratic DILI. Because of its low incidence, idiosyncratic DILI cannot be detected in preclinical testing or clinical trials. $^{9}$

LCZ - the R-enantiomer of cetirizine dihydrochloride, which has favorable pharmacodynamic and pharmacokinetic characteristics - has been demonstrated to be safe and effective for the treatment of allergic diseases, as evidenced by the low number of adverse effects in clinical trials. ${ }^{10,11}$ A similar number of patients 
Moon Chan Jung, et al.
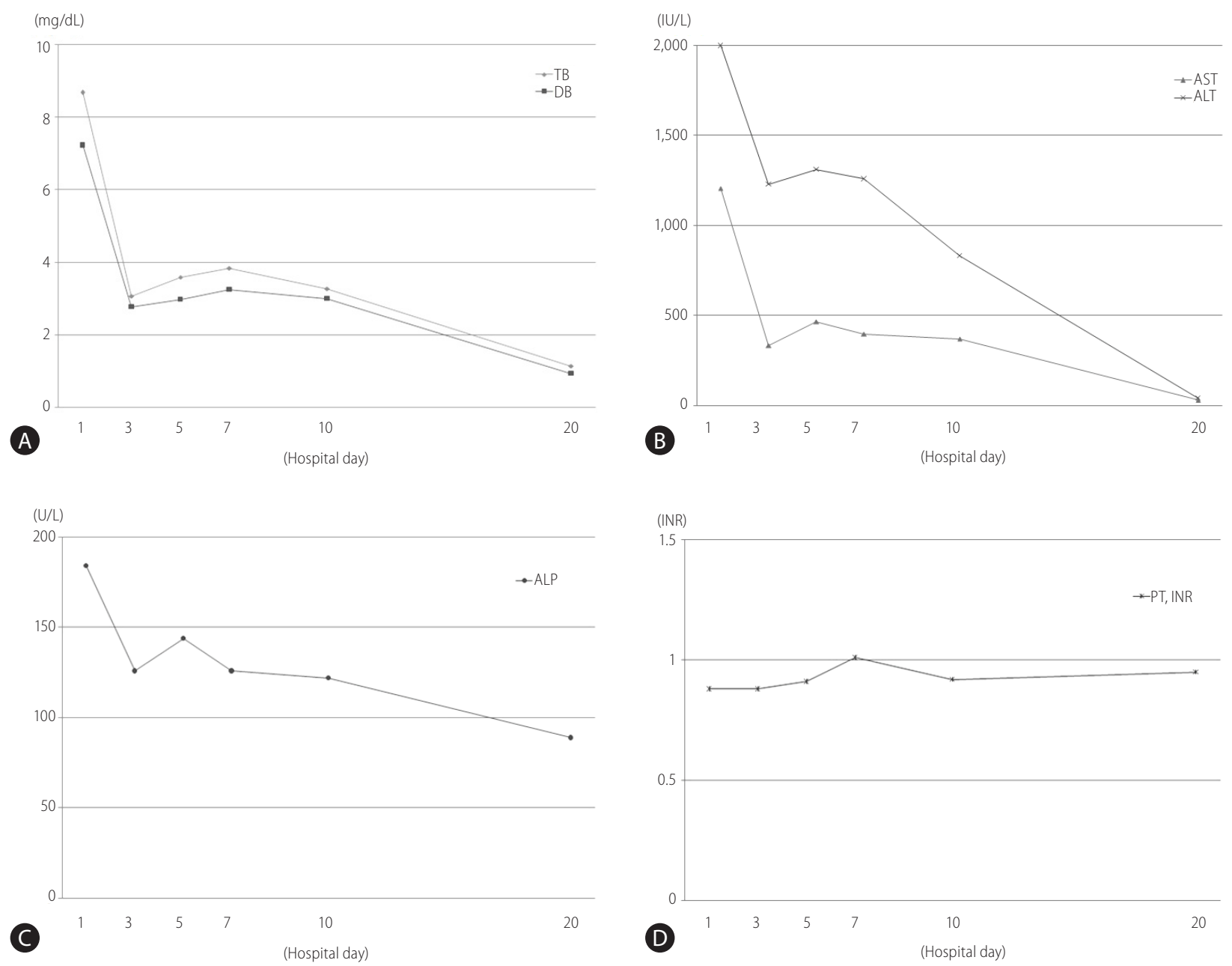

Figure 2. Changes in laboratory parameters over time: (A) total bilirubin (TB) and direct bilirubin (DB), (B) aspartate aminotransferase (AST) and alanine aminotransferase (ALT), (C) alkaline phosphatase (ALP), and (D) prothrombin time (PT).

reported treatment-emergent adverse events with LCZ and placebo. The most common treatment-emergent adverse events are headache (4.6\%), fatigue (1.8\%), and dry throat (1.4\%). One patient in the LCZ group showed cellulitis $(0.4 \%)$ as a serious adverse event. ${ }^{12}$ A previous report of LCZ-induced hepatitis showed less severe hepatic inflammation compared with our case (AST $113 \mathrm{IU} / \mathrm{mL}$ and ALT $115 \mathrm{IU} / \mathrm{mL}$ vs. AST 1,208 IU/mL and ALT 2,043 $\mathrm{IU} / \mathrm{mL}$, respectively). ${ }^{5}$ This difference of severity was originated in which the previous case was detected by routine liver function test after using LCZ only for 2 weeks, but our patient exhibited jaundice after taking LCZ for 2 months. Because hepatotoxicity is not included in the LCZ prescribing information and only one case of LCZ-induced liver injury has been reported, hepatotoxicity was not monitored in our case.

The main mechanism of DILI is an idiosyncratic reaction. Therefore, any drug could cause DILI. Patients with DILI can present with symptoms similar to those of patients with hepatobiliary disease of other causes, and therefore it is important to maintain a high index of clinical suspicion in determining the diagnosis., ${ }^{7,13}$ The clinical presentations of DILI are acute hepatitis and/or cholestasis, although idiosyncratic reactions frequently occur in a background of higher incidence rates of mild asymptomatic liver injury. Our patient did not have a specific past medical history, nor show serologic or radiologic findings. Based on the timing of LCZ administration and the clinical course, we could suspect LCZinduced liver injury. Because the patient conducted liver biopsy and recovered after withdrawal of $L C Z$, we were able to confirm the LCZ-induced liver injury. This case of liver injury was considered an idiosyncratic drug reaction.

Numerous drugs have been reported to cause liver injury, but only one case of LCZ-induced liver injury, which lacked histologic confirmation, has been described. Furthermore, unlike the previ- 
ous case of LCZ-induced liver injury, our patient showed $>20$-fold elevation of serum AST and ALT levels. To our knowledge, this is the first reported case of LCZ-induced liver injury confirmed by liver biopsy.

LCZ is generally well tolerated and widely used, and it has consistently demonstrated high response rates and a favorable sideeffect profile. Although its hepatotoxicity is not established, LCZ could be associated with acute liver injury. In conclusion, LCZ is an effective and well-tolerated drug for the treatment of allergic diseases; however, clinicians should be aware of its potential hepatotoxicity.

\section{Conflicts of Interest}

The authors have no conflicts to disclose.

\section{REFERENCES}

1. Gillard M, Christophe B, Wels B, Peck M, Massingham R, Chatelain P. $\mathrm{H} 1$ antagonists: receptor affinity versus selectivity. Inflamm Res 2003;52(Suppl 1):S49-S50.

2. Grant JA, Riethuisen JM, Moulaert B, DeVos C. A double-blind, randomized, single-dose, crossover comparison of levocetirizine with ebastine, fexofenadine, loratadine, mizolastine, and placebo: suppression of histamine-induced wheal-and-flare response during 24 hours in healthy male subjects. Ann Allergy Asthma Immunol 2002;88:190-197.
3. Xyzal (levocetirizine dihydrochloride) [package insert]. UCB Pharma Inc, Smyrna, Ga; 2008.

4. Maiti R, Rahman J, Jaida J, Allala U, Palani A. Rupatadine and levocetirizine for seasonal allergic rhinitis: a comparative study of efficacy and safety. Arch Otolaryngol Head Neck Surg 2010;136:796-800.

5. Ekiz F, Yuksel I, Ekiz O, Coban S, Basar O, Yüksel O. Levocetirizineinduced hepatotoxicity in a patient with chronic urticaria. Ann Hepatol 2011;10:237-238.

6. Lee WM. Acute liver failure in the United States. Semin Liver Dis 2003:23:217-226.

7. Lewis JH. Drug-induced liver disease. Med Clin North Am 2000;84:1275-1311.

8. Suk KT, Kim DJ, Kim CH, Park SH, Yoon JH, Kim YS, et al. A prospective nationwide study of drug-induced liver injury in Korea. Am J Gastroenterol 2012;107:1380-1387.

9. Kim SH, Naisbitt DJ. Update on advances in research on idiosyncratic drug-induced liver injury. Allergy Asthma Immunol Res 2016;8:3-11.

10. Holgate $S$, Powell $R$, Jenkins $M$, Ali O. A treatment for allergic rhinitis: a view on the role of levocetirizine. Curr Med Res Opin 2005;21:1099-1106.

11. Hair PI, Scott LJ. Levocetirizine: a review of its use in the management of allergic rhinitis and skin allergies. Drugs 2006;66:973-996.

12. Segall N, Gawchik S, Georges G, Haeusler JM. Efficacy and safety of levocetirizine in improving symptoms and health-related quality of life in US adults with seasonal allergic rhinitis: a randomized, placebocontrolled study. Ann Allergy Asthma Immunol 2010;104:259-267.

13. Suk KT, Kim DJ. Drug-induced liver injury: present and future. Clin Mol Hepatol 2012;18:249-257. 